\title{
REAL CANONICAL BINARY SYMMETRIC TRILINEAR FORMS*
}

\section{BY RUFUS OLDENBURGER}

1. Introduction. In treating the equivalence of binary forms of the third degree under non-singular linear transformations it is natural to divide the problem into three parts. These are concerned respectively with the equivalence of forms of the type

$$
a_{i j k} x_{i} y_{j} z_{k}
$$

where

(a) the vectors $\left(x_{1}, x_{2}\right),\left(y_{1}, y_{2}\right),\left(z_{1}, z_{2}\right)$ are distinct;

(b) 2 of the vectors $\left(x_{1}, x_{2}\right),\left(y_{1}, y_{2}\right),\left(z_{1}, z_{2}\right)$ are equal but not equal to the 3 rd;

(c) the vectors $\left(x_{1}, x_{2}\right),\left(y_{1}, y_{2}\right),\left(z_{1}, z_{2}\right)$ are equal.

Dedekind, Schwartz, others, $\uparrow$ and the author have treated the problem of the equivalence of binary trilinear forms in the complex field. Specifically the problem solved was that of determining the conditions on $\left(a_{i j k}\right),\left(a_{p q r}^{\prime}\right)$ such that the form $T=a_{i j k} x_{i} y_{j} z_{k}$ be reducible to the given form $T^{\prime}=a_{p q r}^{\prime} x_{p}{ }^{\prime} y_{q}^{\prime} z_{r}^{\prime}$ under the linear transformations

$$
x_{i}=a_{i p} x_{p}^{\prime}, \quad y_{j}=b_{j q} y_{q}^{\prime}, \quad z_{k}=c_{k r} z_{r}^{\prime},
$$

where $i, j, k, p, q, r=1,2$, and the matrices $\left(a_{i p}\right),\left(b_{j q}\right),\left(c_{k r}\right)$ are non-singular. Recently the author solved this problem of equivalence for the field of reals. $\ddagger$ The theory for case (a) in the field of reals and complexes is hence complete. In the present paper the author treats the equivalence of forms satisfying (b) for the field of reals.

Evidently, the class of forms (c) is contained in the class (b), which in turn is contained in the class (a).

In a new paper, to appear elsewhere, the author has solved the problem of equivalence of the forms in class (c), making

* Presented to the Society, March 26, 1937.

$\dagger$ For a complete list of references see R. Oldenburger, On canonical binary trilinear forms, this Bulletin, vol. 38 (1932), p. 385.

$\ddagger$ R. Oldenburger, Real canonical binary trilinear forms, American Journal of Mathematics, vol. 59 (1937), pp. 427-435. 
use of the results obtained here for class (b), many of which also hold for class (c). Class (c) is simply the class of all binary cubic forms $a_{i j k} x_{i} x_{j} x_{k}$. The theory of forms of classes (a), (b), (c) is thus complete for the field of reals.

The forms in class (b) with real coefficients can always be written as

$$
T=a_{i j k} x_{i} y_{j} y_{k}
$$

where the matrix $A=\left(a_{i j k}\right)$ is symmetric with respect to the $j$ and $k$ indices; $a_{112}=a_{121}, a_{123}=a_{132}, \cdots$. The matrix $A$ is then unique. It is proved in the present paper that $T$ is always equivalent under transformations (1) on the $x$ 's and $y$ 's to one of six canonical forms. These six canonical forms correspond to six canonical pairs of quadratic equations to which pairs of quadratic equations with no linear terms are equivalent.

The treatment involves the use of arithmetic rank invariants, and properties of invariant factors. The technique is essentially different from that used in earlier papers on binary trilinear forms.

2. Definitions. The rank $r_{i}$ of a 3 -way matrix $A=\left(a_{i j k}\right)$, $(i, j, k=1,2)$, and its associated trilinear form $T=a_{i j k} x_{i} y_{j} z_{k}$ is the rank of the 2-way matrix

$$
\left(\begin{array}{llll}
a_{111} & a_{112} & a_{121} & a_{122} \\
a_{211} & a_{212} & a_{221} & a_{222}
\end{array}\right) .
$$

Assume that $a_{i j k},(i, j, k=1,2)$, are not all zero. The 3-way rank $r[j k, i]$ of $A$ and $F$ is defined to be 2 or 1 according as the quantities

$$
\left|\begin{array}{ll}
a_{111} & a_{112} \\
a_{121} & a_{122}
\end{array}\right|,\left|\begin{array}{ll}
a_{211} & a_{212} \\
a_{221} & a_{222}
\end{array}\right|,\left|\begin{array}{ll}
a_{111} & a_{212} \\
a_{121} & a_{222}
\end{array}\right|+\left|\begin{array}{ll}
a_{211} & a_{112} \\
a_{221} & a_{122}
\end{array}\right|
$$

are not all zero or are all zero. These are 3-way determinants with $i$ non-signant.

These ranks have been proved to be invariant under nonsingular linear transformations* on $T$.

3. The Case $r_{i}=r_{j}=r_{k}=2$. We shall need the following lemma:

* R. Oldenburger, Composition and rank of $n$-way mairices and multilinear forms, Annals of Mathematics, vol. 35 (1934), pp. 622-657. 
LEMMA 1. Let $A, B$ be symmetric 2-way matrices of the second order. If $|\rho A+\sigma B| \neq 0$ and $A, B$ are linearly independent, the quadratic form $|\rho A+\sigma B|$ is either indefinite or negative definite.

Under transformation of $A, B$ to

$$
X^{\prime} A X=\left(\begin{array}{ll}
\alpha & 0 \\
0 & \beta
\end{array}\right), \quad X^{\prime} B X=\left(\begin{array}{ll}
a & b \\
b & c
\end{array}\right),
$$

where $X$ is non-singular, the determinant $|\rho A+\sigma B|$ goes into $|X|^{2}|\rho A+\sigma B|$. If $\alpha \beta=0$, then $|\rho A+\sigma B|$ contains the factor $\sigma$. If the determinant of the symmetric matrix of the quadratic form $|\rho A+\sigma B|$ is zero, $|\rho A+\sigma B|$ is equivalent* under nonsingular linear transformation on $\rho, \sigma$ to $\alpha \rho^{\prime 2}$, whence $|\rho A+\sigma B|$ can be written in the factored form $\alpha(g \rho+h \sigma)^{2}$. Hence, if $|\rho A+\sigma B|$ does not factor, $\alpha \beta \neq 0$ and the determinant of the form $|\rho A+\sigma B|$ is $\neq 0$. The matrix of $|\rho A+\sigma B|$ is then regular and non-singular. Then $|\rho A+\sigma B|$ is equivalent to $g \rho^{2}+h \sigma^{2}$. If $g, h$ are of unlike sign the determinant of $|\rho A+\sigma B|$ is $<0$, and $|\rho A+\sigma B|$ factors. If $g, h$ have the same sign this determinant is $>0$. Hence if $|\rho A+\sigma B|$ does not factor, the determinant of $|\rho A+\sigma B|$ is $>0$, whence

$$
(\alpha c-\beta a)^{2}+4 b^{2} \alpha \beta<0 .
$$

If $\alpha \beta>0$ this is not satisfied. Hence if $|\rho A+\sigma B|$ does not factor, $\alpha \beta<0$ and the determinant of $|\rho A+\sigma B|$ is $>0$, whence $|\rho A+\sigma B|$ is negative definite.

If the determinant of $|\rho A+\sigma B|$ is zero, then

$$
(\alpha c-\beta a)^{2}+4 b^{2} \alpha \beta=0 \text {. }
$$

If $\alpha \beta>0,\left(2^{\prime}\right)$ cannot be satisfied unless $b=0$ and $\alpha c-\beta a=0$. Then $A, B$ are linearly dependent. If $\alpha \beta=0,\left(2^{\prime}\right)$ implies that $\alpha c-\beta a=0$, whence, since $\alpha, \beta$ are not both zera, the product. $a c=0$. This implies $\alpha c=\beta a=0$. Hence $|\rho A+\sigma B|=-b^{2} \sigma^{2}$, which is negative definite if $b \neq 0$. As before $b=0$ implies the linear dependence of $A, B$. If $\alpha \beta<0$ the matrix of the form $|\rho A+\sigma B|$ is regular whence $|\rho A+\sigma B|$ is negative definite. If the determinant of $|\rho A+\sigma B|$ is $\neq 0$, and $\alpha \beta=0$, then $|\rho A+\sigma B|$ is indefinite, or is $-b^{2} \sigma^{2}$, which is negative definite.

* L. E. Dickson, Modern Algebraic Theories, p. 88. 
If the rank $r[j k, i]$ of the symmetric form $T=a_{i j k} x_{i} y_{j} y_{k}$, $(i, j, k=1,2)$, is 1 , then ${ }^{*} r_{j}=r_{k}=1$. Hence $r[j k, i]=2$. Let $A=\left(a_{1 j k}\right), B=\left(a_{2 j k}\right)$. The matrices $A, B$ are symmetric matrices. Since the coefficients of $\rho^{2}, \rho \sigma, \sigma^{2}$ in the quadratic form $|\rho A+\sigma B|$ are the quantities (2), the determinant $|\rho A+\sigma B| \not \equiv 0$. Since $r_{i}=2$, the matrices $A, B$ are linearly independent. The matrices $A, B$ hence satisfy the conditions of Lemma 1 .

If the quadratic form $|\rho A+\sigma B|$ is indefinite, it is equivalent under non-singular linear transformations $\dagger$ on $\rho, \sigma$ to $\rho^{\prime 2}-\sigma^{\prime 2}$ which is further equivalent to $\rho^{\prime \prime} \sigma^{\prime \prime}$ under the transformations $\rho^{\prime \prime}=\rho^{\prime}-\sigma^{\prime}, \sigma^{\prime \prime}=\rho^{\prime}+\sigma^{\prime}$. These transformations correspond $\ddagger$ to non-singular linear transformations on $x_{1}, x_{2}$ of $T$, to give a new form $T^{\prime}=a_{i j k}^{\prime} x_{i}^{\prime} y_{j} y_{k}$, for which $\left|\rho^{\prime \prime} A^{\prime}+\sigma^{\prime \prime} B^{\prime}\right|=\rho^{\prime \prime} \sigma^{\prime \prime}$, where $A^{\prime}=\left(a_{1 j k}^{\prime}\right), B^{\prime}=\left(a_{2 j k}^{\prime}\right)$. It follows that $A^{\prime}, B^{\prime}$ are singular. The forms $T_{1}^{\prime}=a_{1 j k}^{\prime} y_{j} y_{k}, T_{2}^{\prime}=a_{2 j k}^{\prime} y_{j} y_{k}$ are equivalent $\S$ to a pair $T_{1}^{\prime \prime}=\alpha y_{1}{ }^{\prime 2}, T_{2}{ }^{\prime \prime}=l\left(y_{1}^{\prime}, y_{2}^{\prime}\right) \cdot m\left(y_{1}^{\prime}, y_{2}^{\prime}\right)$, where $l, m$ are linear in $y_{1}^{\prime}, y_{2}^{\prime}$. Now $l m \neq k y_{1}{ }^{\prime 2}$, since otherwise $r_{i}<2$. It is hence no restriction to take $l \neq k y_{1}^{\prime}$. Let $y_{1}^{\prime \prime}=y_{1}^{\prime}, y_{2}^{\prime \prime}=l\left(y_{1}^{\prime}, y_{2}^{\prime}\right)$, whence the pair $T_{2}^{\prime \prime}, T_{2}{ }^{\prime \prime}$ transforms non-singularly into $T_{1}{ }^{\prime \prime}=\alpha y_{1}^{\prime \prime 2}$, $T_{2}^{\prime \prime \prime}=y_{2}^{\prime \prime} n\left(y_{1}^{\prime \prime}, y_{2}^{\prime \prime}\right)$, where $n$ is linear in $y_{1}^{\prime \prime}, y_{2}^{\prime \prime}$. Since $T_{2}^{\prime \prime \prime}$ is singular, $n\left(y_{1}^{\prime \prime}, y_{2}^{\prime \prime}\right)=\beta y_{2}^{\prime \prime}$ for some $\beta$. Since $r_{i}=2 ; \alpha, \beta \neq 0$. Let $x_{1}^{\prime \prime}=\alpha x_{1}^{\prime}, x_{2}^{\prime \prime}=\beta x_{2}^{\prime}$. Then $x_{1}^{\prime} T_{1}^{\prime \prime \prime}+x_{2}^{\prime} T_{2}^{\prime \prime \prime}$ transforms into the canonical form

$$
R=x_{1} y_{1}^{2}+x_{2} y_{2}^{2},
$$

where the primes on the variables have been dropped for the sake of simplicity.

If $|\rho A+\sigma B|$ is a negative definite quadratic form of rank 1 , it is equivalent $\|$ to $-\sigma^{\prime 2}$. This corresponds to the transition from $T$ to $T^{\prime}=a_{i j k}^{\prime} x_{i}^{\prime} y_{j} y_{k}$, where $\left|a_{1 j k}\right|=0$, and

$$
\left|\begin{array}{ll}
a_{111}^{\prime} & a_{212}^{\prime} \\
a_{121}^{\prime} & a_{222}^{\prime}
\end{array}\right|+\left|\begin{array}{ll}
a_{211}^{\prime} & a_{112}^{\prime} \\
a_{221}^{\prime} & a_{122}^{\prime}
\end{array}\right|=0 .
$$

* R. Oldenburger, op. cit., p. 649.

$\dagger$ L. E. Dickson, Modern Algebraic Theories, p. 72.

$\ddagger$ R. Oldenburger, Non-singular multilinear forms and certain p-way matrix factorizations, Transactions of this Society, vol. 39 (1936), p. 432.

§ L. E. Dickson, op. cit., p. 69.

|| L. E. Dickson, op. cit., p. 72. 
The pair of quadratic forms $a_{1 j k}^{\prime} y_{j} y_{k}, a_{2 j k}^{\prime} y_{j} y_{k}$ is now equivalent to a pair $\alpha y_{1}^{\prime 2}, a y_{1}^{\prime 2}+2 b y_{1}^{\prime} y_{2}^{\prime}+c y_{2}^{\prime 2}$, for which

$$
\left|\rho^{\prime}\left(\begin{array}{ll}
\alpha & 0 \\
0 & 0
\end{array}\right)+\sigma^{\prime}\left(\begin{array}{ll}
a & b \\
b & c
\end{array}\right)\right|=-K^{2} \sigma^{\prime 2},
$$

where $K$ is the modulus of transformation. The analogue of (3) gives $\alpha c=0$, whence, since $\alpha \neq 0$, we have $c=0$. Now $b \neq 0$, since $r_{i}=2$. If we write

$$
x_{1}^{\prime \prime}=\alpha x_{1}^{\prime}, \quad x_{2}^{\prime \prime}=x_{2}^{\prime}, \quad y_{1}^{\prime \prime}=y_{1}^{\prime}, \quad y_{2}^{\prime \prime}=\frac{a}{2} y_{1}^{\prime}+b y_{2}^{\prime},
$$

the form $x_{1}^{\prime} \alpha y_{1}^{\prime 2}+x_{2}^{\prime}\left(a y_{1}^{\prime 2}+2 b y_{1}^{\prime} y_{2}^{\prime}\right)$ transforms into the canonical form

$$
L=x_{1} y_{1}{ }^{2}+2 x_{2} y_{1} y_{2},
$$

where the primes have been dropped.

To treat the class of forms $T$ for which $|\rho A+\sigma B|$ does not factor in the field of reals we shall need another lemma:

Lemma 2. A pair $P$ of quadratic forms $x^{2}-y^{2}$, ex $x^{2}+2 f x y+g y^{2}$ is equivalent under non-singular linear transformations to the pair $x^{2}-y^{2}, 2 x y$ if and only if the characteristic determinant

of $P$ is $-\rho^{2}-\sigma^{2}$.

$$
\left|\rho\left(\begin{array}{rr}
1 & 0 \\
0 & -1
\end{array}\right)+\sigma\left(\begin{array}{ll}
e & f \\
f & g
\end{array}\right)\right|
$$

The necessity is evident.* To prove the sufficiency assume that the characteristic determinant of $P$ is $-\rho^{2}-\sigma^{2}$. Then $e, f, g$ satisfy the relations

$$
g=e, \quad f^{2}-e^{2}=1 .
$$

An elementary computation reveals that the matrix $X$ of the most general non-singular linear transformation leaving $x^{2}-y^{2}$ invariant in the sense that

$$
X^{\prime}\left(\begin{array}{rr}
1 & 0 \\
0 & -1
\end{array}\right) X=\left(\begin{array}{rr}
1 & 0 \\
0 & -1
\end{array}\right)
$$

where $X^{\prime}$ is $X$-transpose, is of the form

* L. E. Dickson, op. cit., p. 97. 


$$
X=J\left(\begin{array}{ll}
a & b \\
b & a
\end{array}\right)
$$

where

$$
J=\left(\begin{array}{ll}
1 & 0 \\
0 & 1
\end{array}\right) \quad \text { or } \quad\left(\begin{array}{rr}
1 & 0 \\
0 & -1
\end{array}\right)
$$

and

$$
a^{2}-b^{2}=1
$$

If the pairs of forms of the theorem are equivalent there exists a matrix $X$ of the above form such that

$$
X^{\prime}\left(\begin{array}{ll}
0 & 1 \\
1 & 0
\end{array}\right) X=E
$$

where

$$
E=\left(\begin{array}{ll}
e & f \\
f & g
\end{array}\right)
$$

Since

$$
J^{\prime}\left(\begin{array}{ll}
0 & 1 \\
1 & 0
\end{array}\right) J= \pm\left(\begin{array}{ll}
0 & 1 \\
1 & 0
\end{array}\right)
$$

it follows that

$$
X^{\prime}\left(\begin{array}{ll}
0 & 1 \\
1 & 0
\end{array}\right) X \equiv \pm\left(\begin{array}{ll}
2 a b & a^{2}+b^{2} \\
a^{2}+b^{2} & 2 a b
\end{array}\right) .
$$

We shall show that it is always possible to choose the matrix (5) equal to $E$, where the elements of $E$ satisfy (4). Now $E$ is of the form

or

$$
E_{1}=\left(\begin{array}{ll}
e & \left(1+e^{2}\right)^{1 / 2} \\
\left(1+e^{2}\right)^{1 / 2} & e
\end{array}\right),
$$

$$
E_{2}=\left(\begin{array}{cc}
e & -\left(1+e^{2}\right)^{1 / 2} \\
-\left(1+e^{2}\right)^{1 / 2} & e
\end{array}\right) \text {. }
$$

Take $e \neq 0$. If $E=E_{1}$ equate $E_{1}$ and (5) with the + sign: if $E=E_{2}$ equate $E_{2}$ and (5) with the - sign. We obtain 


$$
e= \pm 2 a b, \quad\left(1+e^{2}\right)^{1 / 2}=a^{2}+b^{2}
$$

We obtain as real solutions of (6)

$$
b=\left(\frac{\left(1+e^{2}\right)^{1 / 2}-1}{2}\right)^{1 / 2} ; \quad a= \pm \frac{e}{2 b} ;
$$

which identically satisfy the condition $a^{2}-b^{2}=1$. If $e=0$,

$$
E_{1} \equiv\left(\begin{array}{ll}
0 & 1 \\
1 & 0
\end{array}\right), \quad E_{2} \equiv\left(\begin{array}{rr}
0 & -1 \\
-1 & 0
\end{array}\right) .
$$

In the latter case take the $-\operatorname{sign}$ in (5), and $a=0, b=1$. Then $(5) \equiv E_{2}$.

We shall now show by Lemmas 1 and 2 that $T=a_{i j k} x_{i} y_{j} y_{k}$, where $|\rho A+\sigma B|$ does not factor, is equivalent to a canonical form of the type

$$
M=x_{1}\left(y_{1}^{2}-y_{2}^{2}\right)+2 x_{2} y_{1} y_{2} .
$$

By Lemma $1\left|\rho\left(a_{1 j k}\right)+\sigma\left(a_{2 j k}\right)\right|$ is equivalent under non-singular linear transformations on $\rho, \sigma$ to $-\rho^{2}-\sigma^{2}$, since it is negative definite. This corresponds to a non-singular linear transformation* on $x_{1}, x_{2}$ of $T$ to give a new form $T^{\prime}=a_{i j k}^{\prime} x_{i}^{\prime} y_{j} y_{k}$ such that

$$
\left|\rho\left(a_{1 j k}^{\prime}\right)+\sigma\left(a_{2 j k}^{\prime}\right)\right|=-\rho^{2}-\sigma^{2} .
$$

Since $\left|a_{1 j k}^{\prime}\right|=-1$, the form $a_{1 j k}^{\prime} y_{j} y_{k}$ is equivalent under orthogonal transformations $\dagger$ on the $y^{\prime}$ s to $\alpha y_{1}^{\prime 2}-y_{2}^{\prime 2} / \alpha, \alpha>0$, and $a_{2 j k}^{\prime} y_{j} y_{k}$ transforms simultaneously to a new form $T_{2}^{\prime}$. This pair of forms is further equivalent under the transformations $y_{1}^{\prime \prime}=\alpha^{1 / 2} y_{1}^{\prime}, y_{2}^{\prime \prime}=y_{2}^{\prime} / \alpha^{1 / 2}$ of determinant unity to a pair $C_{3}=y_{1}^{\prime \prime 2}$ $-y_{2}^{\prime \prime 2}, T_{2}^{\prime \prime}$ with matrices which will be denoted by $A^{\prime}, B^{\prime}$ respectively. Now $\left|\rho A^{\prime}+\sigma B^{\prime}\right|=-\rho^{2}-\sigma^{2}$. By Lemma 2, $x_{1}^{\prime} C_{3}$ $+x_{2}^{\prime} T_{2}^{\prime \prime}$ is equivalent to $M$.

Since the rank and index of the quadratic form $\mid \rho\left(a_{1 j k}\right)$ $+\left(a_{2 j k}\right) \mid$ are invariant under non-singular linear transformations on $T=a_{i j k} x_{i} y_{j} y_{k}$, no two in the set of canonical forms $R, L, M$ are equivalent.

* See R. Oldenburger, Non-singular multilinear forms and certain p-way matrix factorizations, loc. cit.

† L. E. Dickson, op, cit., p. 74. 
4. The Cases where $\left(r_{i}, r_{j}, r_{k}\right) \neq(2,2,2)$. By symmetry $r_{j}=r_{k}$. If $r_{i}=2, r_{j}=r_{k}=1$, then* $r[i j, k]=r[k j, i]=r[i k, j]=1$, which, as is easy to show, implies that $r_{i}=1$, giving a contradiction. If $r_{i}=1, r_{j}=r_{k}=2$, then $T=a_{i j k} x_{i} y_{j} y_{k}$ is equivalent at once to $P=x_{1}\left(y_{1}^{2}+y_{2}^{2}\right)$ or $Q=x_{2}\left(y_{1}^{2}-y_{2}^{2}\right)$. In the first case $\mid \rho\left(a_{1 j k}\right)$ $+\sigma\left(a_{2 j k}\right) \mid$ is positive definite, in the latter case negative definite. If $r_{i}=r_{j}=r_{k}=1, T$ is equivalent to $x_{1} y_{1}^{2}$. Evidently $P, Q$ are not equivalent.

5. Results. By the invariance of rank it follows that the canonical forms obtained are not equivalent. We have proved this theorem:

THEOREM. Two binary symmetric forms $T=a_{i j k} x_{i} y_{j} y_{k}$ and $T^{\prime}=a_{i j k}^{\prime} x_{i}^{\prime} y_{j}^{\prime} y_{k}^{\prime}$ are equivalent if and only if they have the same set of ranks $\left[r_{i}, r_{j}, r_{k}\right]$, and the quadratic forms $\left|\rho\left(a_{1 j k}\right)+\sigma\left(a_{2 j k}\right)\right|$, $\left|\rho\left(a_{1 j k}^{\prime}\right)+\sigma\left(a_{2 j k}^{\prime}\right)\right|$ have the same rank and index.

It follows from the theory that a pair of quadratic equations with no linear terms is equivalent to one of the canonical pairs

$$
\begin{gathered}
{\left[x^{2}=a, y^{2}=b\right],\left[x^{2}=a, x y=b\right],\left[x^{2}-y^{2}=a, x y=b\right]} \\
{\left[x^{2}+y^{2}=a, 0=0\right],\left[x^{2}-y^{2}=a, 0=0\right]}
\end{gathered}
$$

or $\left[x^{2}=a, 0=0\right]$, where the roots of the original pair are linear in the roots of the canonical pair.

Armour Institute of Technology

The Institute for Advanced Study

* R. Oldenburger, Composition and rank of $n$-way matrices and multilinear forms, Annals of Mathematics, vol. 35 (1934), p. 641. 